\title{
Nouveaux espaces numériques du dessin
}

\section{Alexandre Clérisse}

\section{OpenEdition}

\section{Journals}

Édition électronique

URL : http://journals.openedition.org/genesis/1698

DOI : 10.4000/genesis. 1698

ISSN : 2268-1590

\section{Éditeur :}

Presses universitaires de Paris Sorbonne (PUPS), Société internationale de génétique artistique littéraire et scientifique (SIGALES)

\section{Édition imprimée}

Date de publication : 12 décembre 2016

Pagination : 123-131

ISBN : 9791023105490

ISSN : 1167-5101

\section{Référence électronique}

Alexandre Clérisse, « Nouveaux espaces numériques du dessin », Genesis [En ligne], 43 | 2016, mis en ligne le 29 octobre 2017, consulté le 19 avril 2019. URL : http://journals.openedition.org/genesis/1698 ; DOI : 10.4000/genesis. 1698

Ce document a été généré automatiquement le 19 avril 2019.

Tous droits réservés 


\title{
Nouveaux espaces numériques $d u$ dessin
}

\author{
Alexandre Clérisse
}

Dans le dessin dit vectoriel, il n'y a pas de « trait » mais des points que l'on relie entre eux par des droites ou des courbes de Bézier et auxquels on peut appliquer différentes transformations (homothéties, rotations, écrasement, mise à l'échelle, extrusion, inclinaison, effet miroir, dégradé de formes, etc.), comme des formes molles modifiables à volonté, sans jamais perdre de qualité. Contrairement aux images dites matricielles, constituées de pixels, le vectoriel permet de zoomer dans le dessin au plus près et d'être toujours net. Cela reviendrait à dessiner avec un fil dont on peut arranger les contours. Mais ce fil peut se remplir de n'importe quelle couleur ou de n'importe quel motif, et devient ensuite une forme libre, comme un morceau de papier découpé. Une fois que l'on assemble ces papiers découpés, chaque forme peut être déformée indépendamment. Cela change totalement la perception du dessin, car on se rapproche alors du modelage et du découpage (fig. 1).

\begin{tabular}{|c|c|c|}
\hline -Image & 2000000900004E600000058FC7D2F181.wmf & Image \\
\hline 2000000 & 058F73887CBE.wmf & Image \\
\hline 2000000 & 058F43D4BC3C.wmf & \\
\hline Image & 2000000900004EF9000005A9BF5E5C7F.wmf & Image \\
\hline 2000000 & 058F22F97DF6.wmf & Image \\
\hline 2000000 & 05DBBDB40984.wmf & \\
\hline nage & 20000009000051F3000005C21F919C9B.wmf & Image \\
\hline
\end{tabular}
600A10C2DB.wmf

Fig. 1

2 L'espace de travail se présente comme une immense feuille au centre de laquelle se trouve le dessin final, tout le reste étant constitué des marges infinies où peuvent être 
importées d'autres formes, personnages déjà créés dans diverses postures, arbres multiples ou motifs variables (fig. 2 et 3 ).

Image 2000000900011F1B0000014BD4B66FFC.wmf

Fig. 2: Illustrator : exemple d'un dispositif de travail

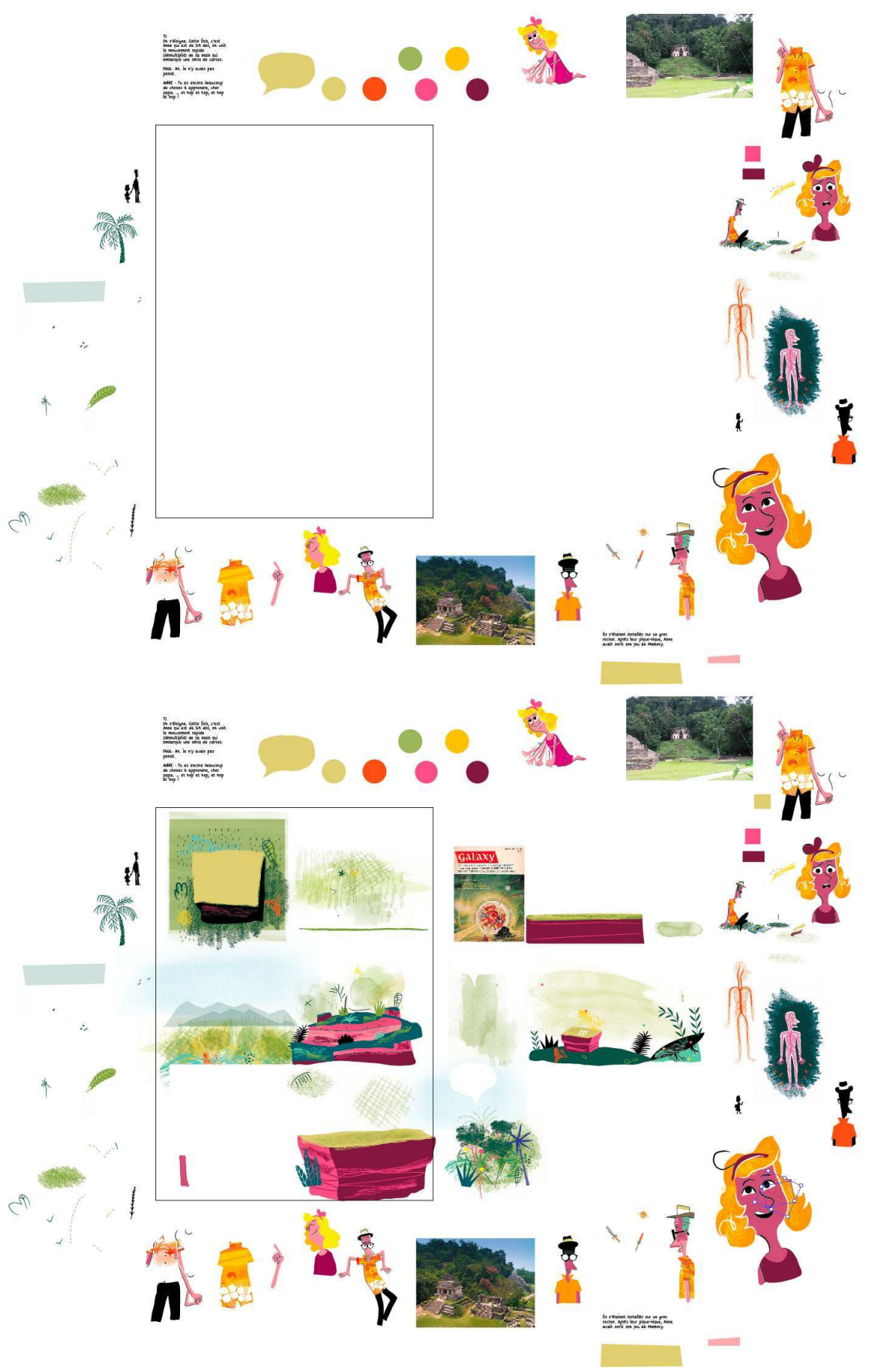




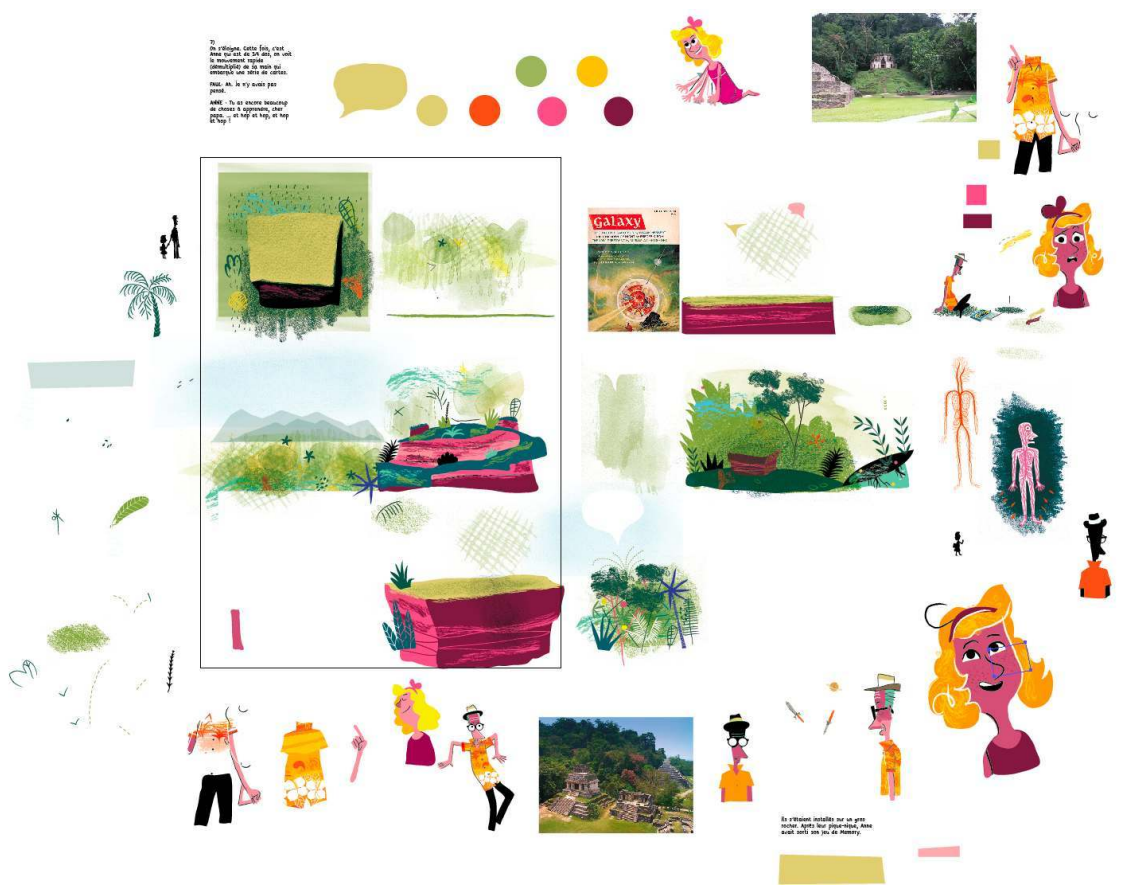




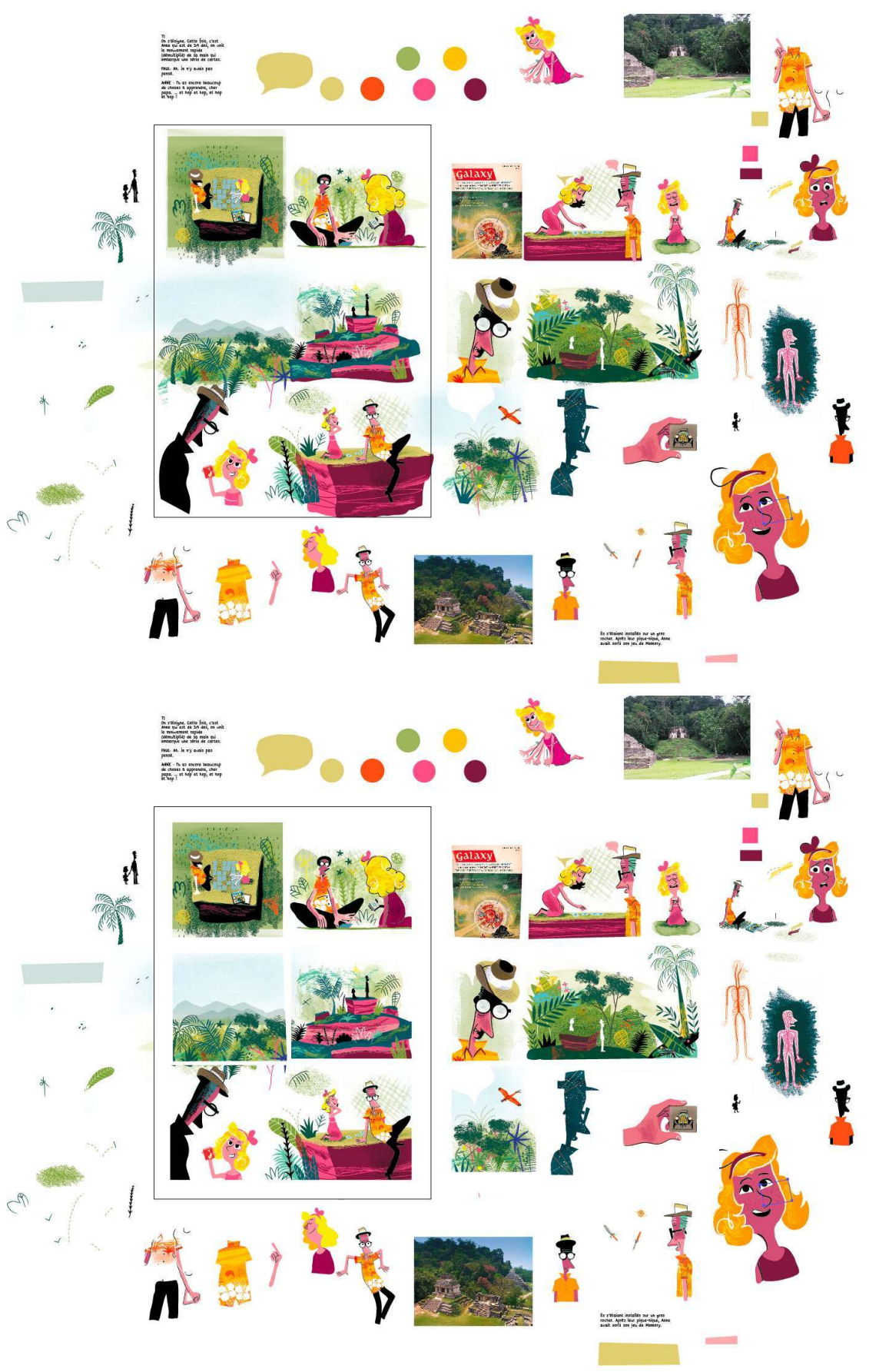




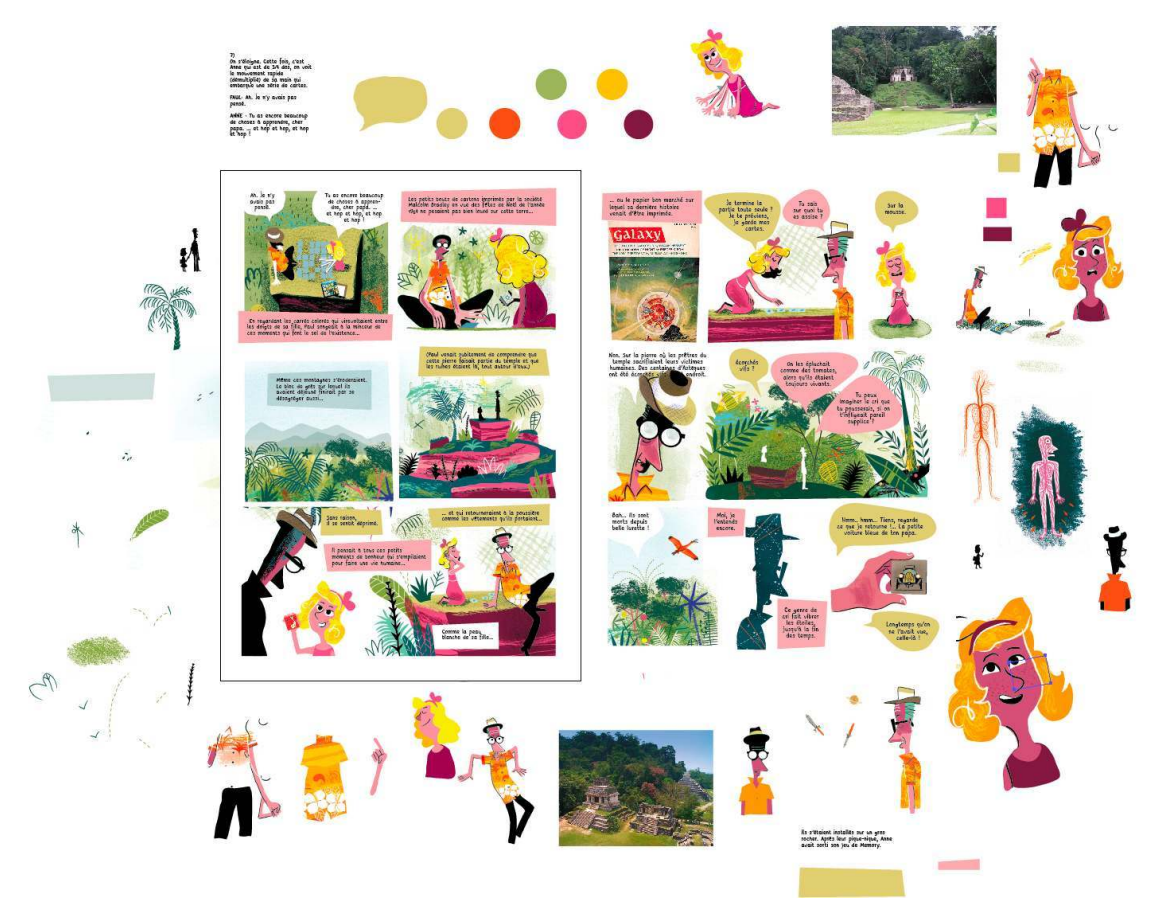

Fig. 3 : Captures numériques de l'écran de travail créent des marionnettes de leurs personnages dont chaque partie reste mobile, mais cette technique est rarement utilisée en bande dessinée, les auteurs préférant les images matricielles qui se rapprochent plus du travail pictural.

Personnellement, mon approche du dessin vectoriel s'est faite empiriquement et encore aujourd'hui, je ne maîtrise pas toutes les possibilités de cette technique. Elle m'a d'abord paru froide et incompréhensible lorsque j'y ai fait mes premiers pas. Comment doit-on penser le dessin, en commençant par le trait ou par la forme? Le travail, avec des éléments purement graphiques, dont la simplicité et l'épure des formes primitives (carrés, cercles...), permet de créer d'innombrables combinaisons : sans doute est-ce un élément de réponse. Par ailleurs, avant de me plonger dans le vectoriel, mon expérimentation en bande dessinée se tournait plutôt alors vers la peinture et des techniques lourdes et laborieuses qui figeaient mon dessin: le temps passé sur mes images me faisait oublier le fil de la narration. Sachant qu'un lecteur passe quelques secondes sur une case, l'écart entre le temps de travail et le temps de lecture peut nuire au dynamisme d'une histoire.

5 J'étais alors fasciné par les travaux de l'Oubapo et de Chris Ware, leur rapport à l'écriture et à la décomposition du mouvement. Durant mes études à l'ESI d'Angoulême, Thierry Smolderen nous a lancé le défi d'un feuilleton en improvisation, dans l'esprit du « Garage Hermétique » de Mœbius (fig. 4).

Image 200000090001124F000001970A1263B9.wmf Fig. 4 :

6 Je souhaitais mettre en scène une histoire en couleur directe, mais dont la rapidité d'exécution me permettrait de distendre le temps et de répondre à la contrainte. Plus tard, pour L'Été Diabolik ${ }^{1}$, j'ai donc créé la marionnette de mon personnage (fig. 5). 


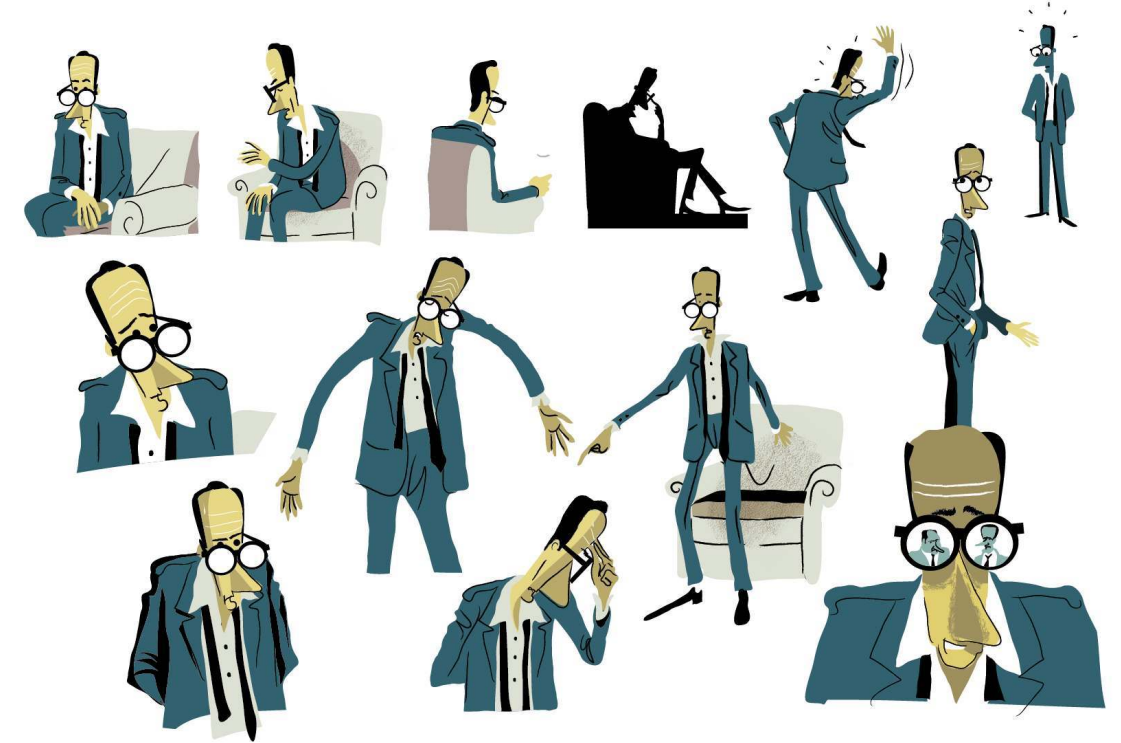

Fig. 5 :

7 En appuyant sur une touche, je le duplique dans la case suivante. Il suffit alors de bouger un bras, déformer quelques points, varier une couleur, pour mettre son personnage en mouvement. D'un seul coup, la vitesse d'exécution prend la forme d'une écriture et la narration se fluidifie.

8 Le dessin se compose alors comme un jeu de construction ou un puzzle dans lequel on manipule les pièces jusqu'à trouver la combinaison parfaite, celle qui servira le mieux ce que l'on veut raconter (fig. 6).

Image 20000009000065FD0000060E20C7BFDF.wmf

Image

2000000900006AA700000360859CDC3C.wmf 


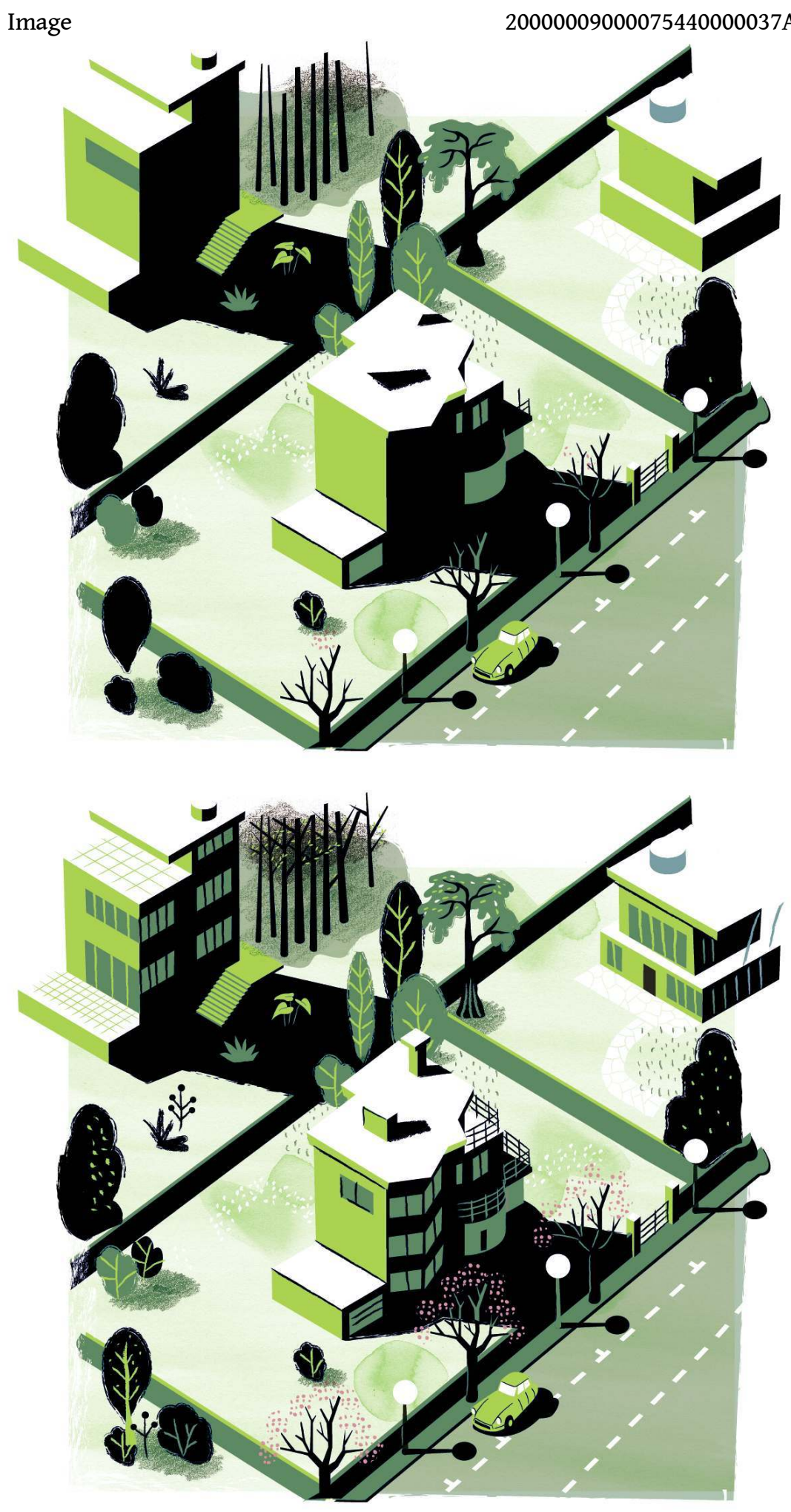

Fig. 6:

9 J'ai ainsi trouvé le rendu coloré que je souhaitais et la vivacité d'écriture qui me manquait. En opposition au dessin en pixels, le pouvoir de revenir en arrière n'est pas celui de pouvoir refaire à l'infini le trait parfait, mais plutôt d'être dans une création en mouvement. Les opposants au dessin numérique diront qu'il n'y a plus d'accident créatif 
possible; que le travail d'équilibriste du dessinateur fait la force du «vrai » dessin. Certaines erreurs sur papier se corrigent (il suffit de voir les collages et la gouache blanche sur nombre de pages de grands maîtres de la bande dessinée), mais parfois il faut tout recommencer, c'est ce qui donne du piment aux outils dit «traditionnels». De l'accident peuvent naître des choses merveilleuses. Mais le pouvoir de revenir une seconde en arrière amène d'autres questions dans la création : quand arrêter de travailler son image? Qu'est-ce qu'un dessin parfait? La machine ne fait pas de tache d'encre providentielle, mais parfois des vecteurs se croisent, créant une nouvelle forme, ou deux formes déstructurées dans le coin d'une marge font apparaître une nouvelle vision.

10 Autre hasard magique de cet outil, lorsque l'on sélectionne tous les points d'encrage d'un dessin, on obtient une constellation colorée abstraite de son image, apportant encore une nouvelle vision du dessin, source de trouvailles visuelles (fig. 7). 

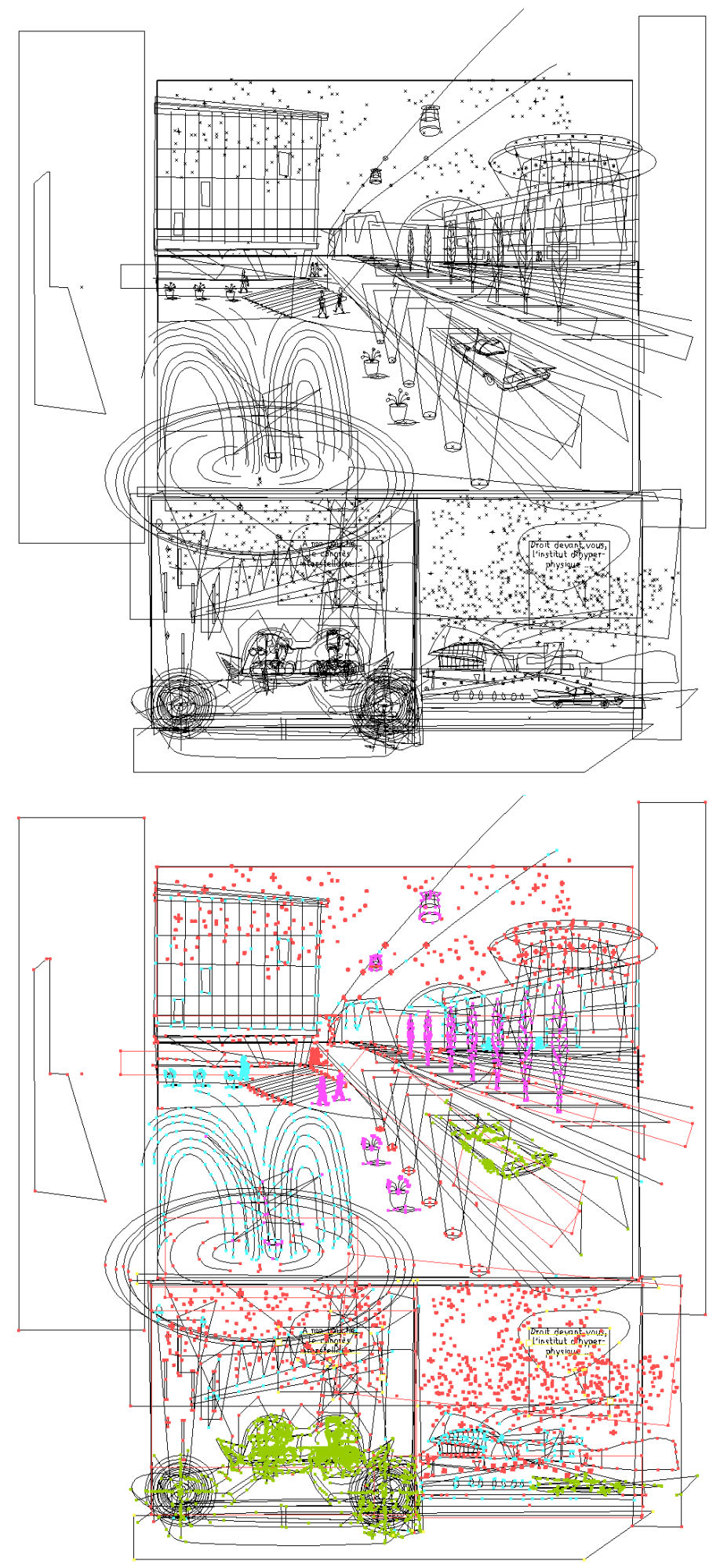

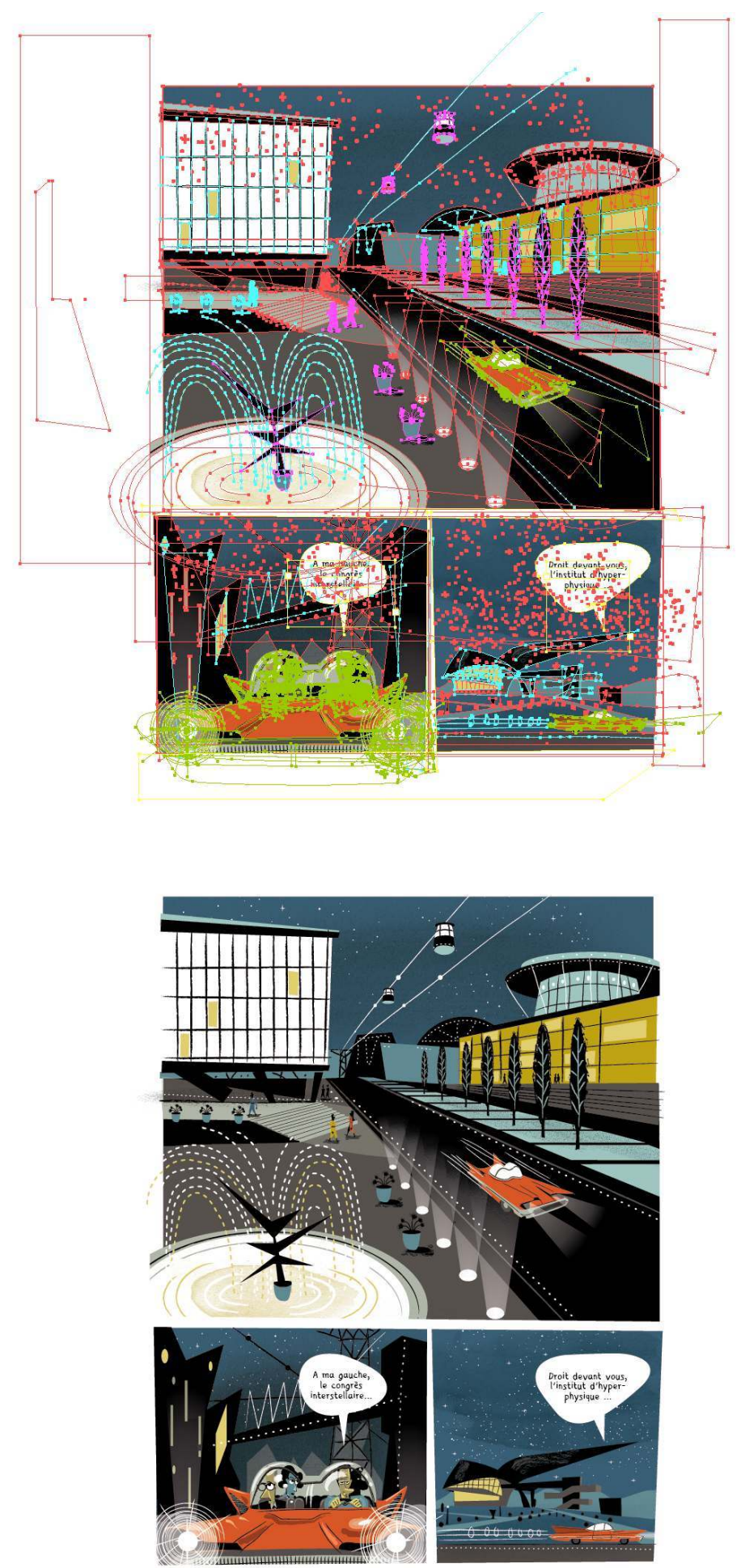

Fig. 7 :

11 Récemment, j'ai tenté de reproduire en peinture sur papier des images que j'ai réalisées en vectoriel (fig. 8). 

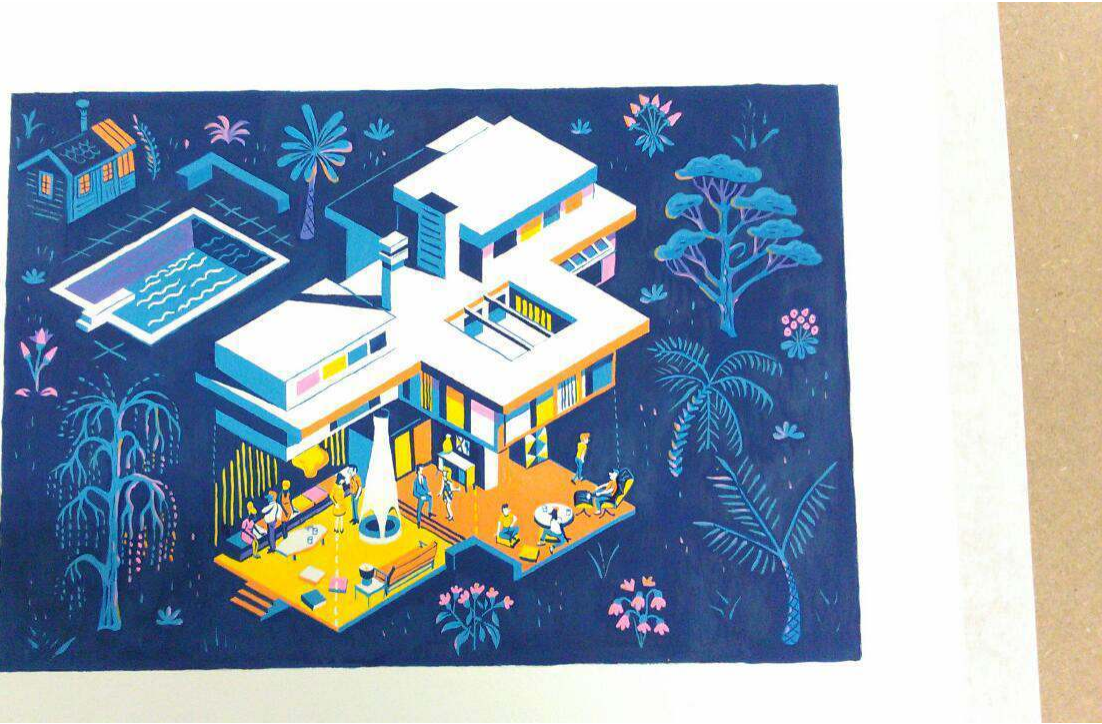

Fig. 8 : Image numérique puis copie picturale de l'image numérique

Cet exercice me permet de mieux comprendre la façon dont je crée ces images et comment je pourrais rajouter de la spontanéité dans mon travail informatique. Je me suis ainsi rendu compte que certaines parties de mes dessins manquaient de spontanéité, car très longues à exécuter sur papier, à l'inverse, d'autres éléments, très longs et laborieux à réaliser sur écran, peuvent être faits d'un geste très rapide sur papier. J'essaie également d'introduire des textures picturales, des taches, des griffonnages, des lavis... qui se colorient dans mon logiciel et deviennent eux aussi des éléments mobiles de mon langage (fig. 9).

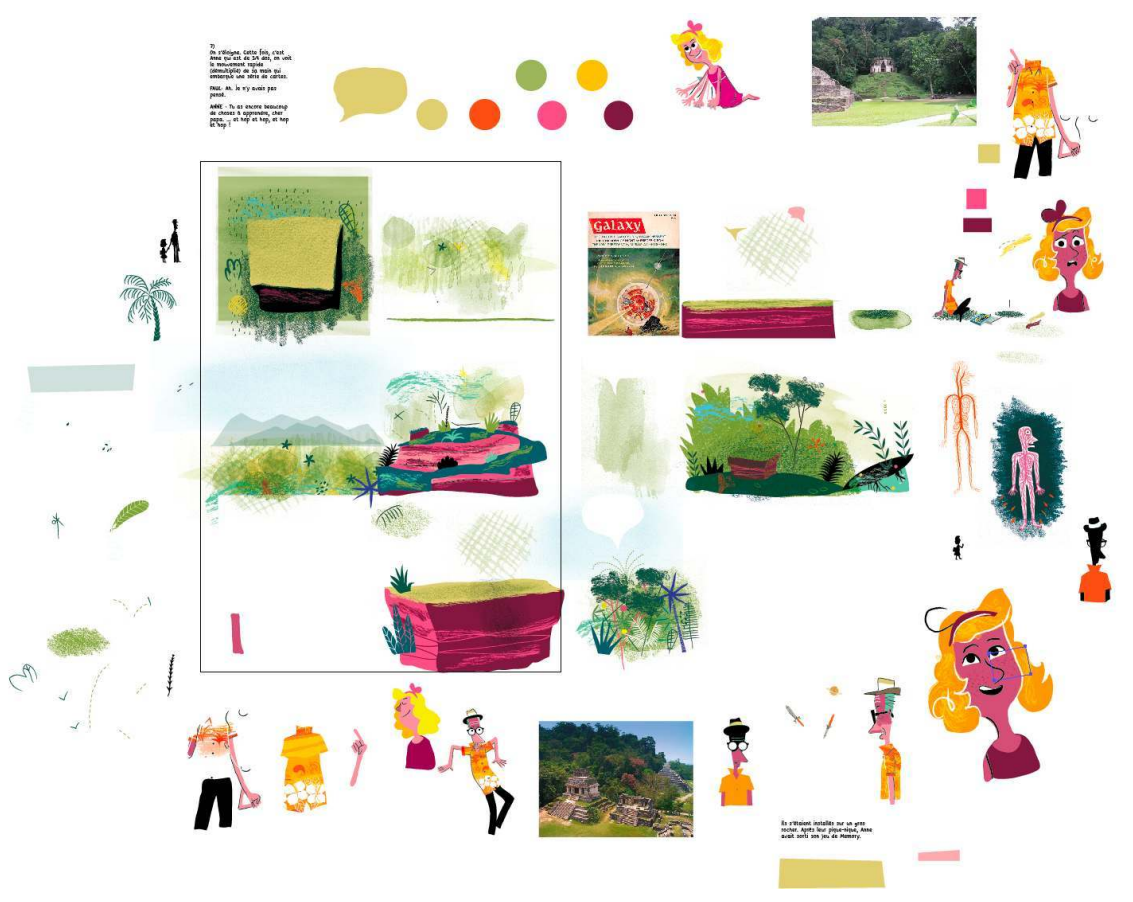

Fig. 9

Je n'en suis encore qu'aux balbutiements de mon langage avec l'outil vectoriel, je dois encore lutter avec la froideur et la fatigue visuelle de l'écran, mais je pense que j'aurai 
toujours besoin de faire ces allers-retours entre l'écran et le papier pour enrichir mon écriture.

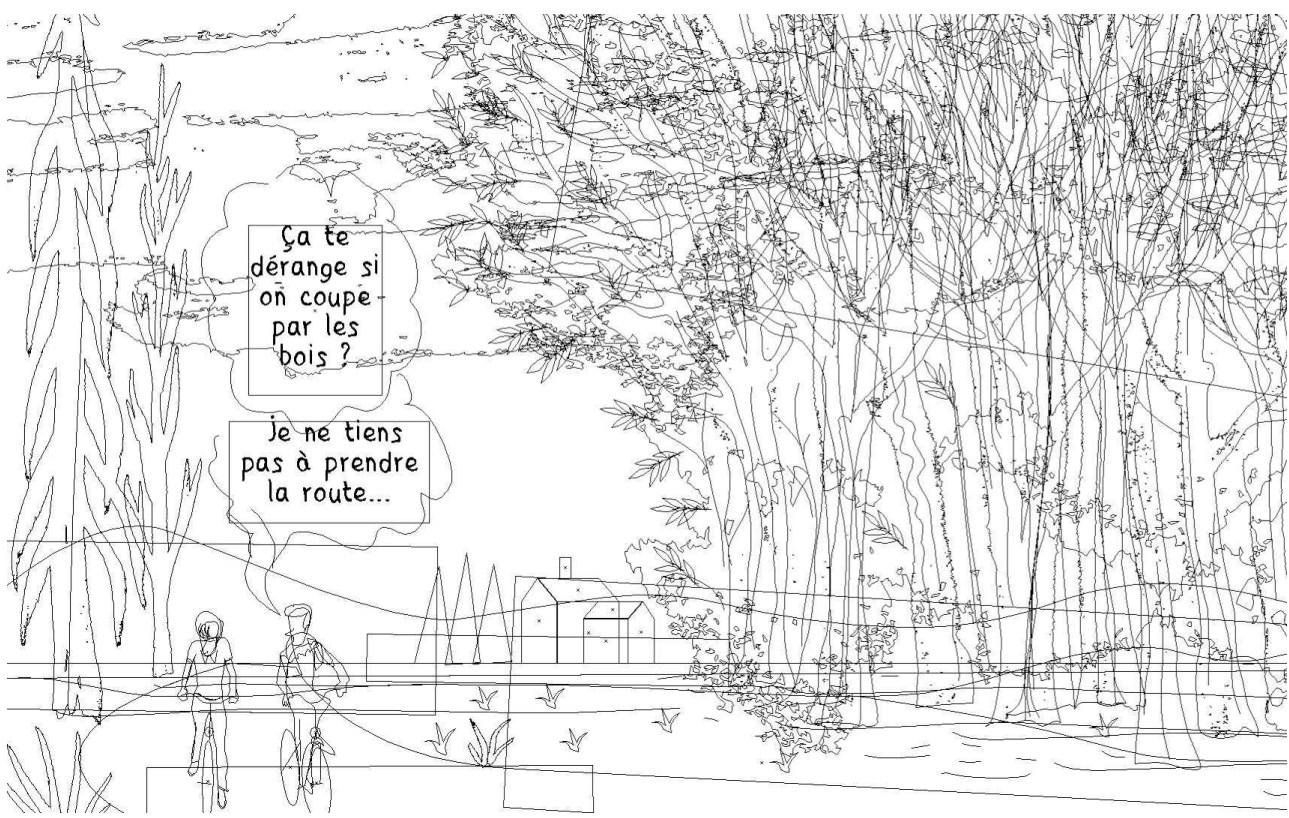

Fig. 10

\section{NOTES}

1. Thierry Smolderen et Alexandre Clarisse, L'Été diabolik, Dargaud, janvier 2016.

\section{RÉSUMÉS}

Alexandre Clérisse propose ici une explication du processus du dessin sous Illustrator, un logiciel vectoriel qui permet une composition intégrale de la case, de la planche et de l'album par ordinateur. Ceci n'interdit pas, en amont, un dialogue avec le dessin traditionnel au profit d'une très grande variété de formes, d'une gamme complexe de signes graphiques et d'une remarquable liberté de création.

Alexandre Clérisse proposes here an explanation of the process of drawing using Illustrator, a vector graphics software that allows an integral composition of the frame, the plate and the album with a computer. This does not prevent a previous dialogue with traditional drawing to the benefit of a great variety of forms, a complex range of graphic signs and a remarkable freedom of composition. 
Alexandre Clérisse propone aquí una explicación del proceso de dibujo con Illustrator, un programa vectorial que permite realizar, a través del ordenador, una composición integral del cuadro, la lámina y la historieta completa. Este proceso no impide un diálogo previo con el dibujo tradicional, en beneficio de una gran variedad de formas, una gama compleja de signos gráficos y una notable libertad de creación.

Alexandre Clérisse ci propone una spiegazione del processo di disegno con Illustrator, un programma vettoriale che permette una composizione integrale della vignetta, della tavola e dell'albo attraverso il computer. Ciò non impedisce di dialogare, a monte, con il disegno tradizionale, pur continuan do ad approfittare di una varietà molto ampia di forme, di una gamma complessa di segni grafici e di una notevole libertà di creazione.

Alexandre Clérisse versucht hier eine Explikation des Prozesses der Zeichnung im Rahmen von „Illustrator“, einer vektoriellen Software, die eine vollständige Komposition von Feld, Bildtafel und Album am Computer ermöglicht. Das hindert nicht daran, einen Dialog mit der traditionellen Zeichnung zu führen, was eine sehr große Vielfalt an Formen begünstigt, sowie eine komplexe Palette graphischer Zeichen und eine bemerkenswerte Freiheit des Kreationsprozesses.

\section{AUTEUR}

\section{ALEXANDRE CLÉRISSE}

Venu du monde de l'infographie, du design et de l'illustration, Alexandre Clérisse (né en 1980) a fait l'expérience du fanzine et du travail en revue à partir de ses années de formation à l'École européenne supérieure de l'image à Angoulême avant de s'engager, à partir de 2006, dans des travaux de plus grande ampleur. Sa collaboration avec le scénariste Thierry Smolderen a permis de croiser les images et les imaginaires : dans Souvenirs de l'Empire de l'Atome et L'Été Diabolik (Dargaud, respectivement 2013 et 2016), il continue de perfectionner une écriture graphique vectorielle fascinante, où les possibilités offertes par le logiciel influent sur les choix de mise en image. 